\title{
Die Verständlichkeit von Rechtstexten und interaktionsorientierter Ansatz
}

\begin{abstract}
A lack of consent about legal terms and concepts is not just a communication problem, but may also be viewed as a problem of the definition of legal terms and concepts.

This paper starts by discussing several reader text models, and goes on to introducing the concept of the open text. This concept helps to analyse contexts in which, apart from texts, the definitions of legal concepts and terms (for example "legal text", " legal language" or "legal comprehensibility") are generated during complex, dynamic and ideally democratic interactions. A unilateral act of definition does not only assign certain meanings, rules of interpretation, or authorisation of executive action to legal terms, but also ademocratic assumptions about political participation. In this constellation, individuals will always be subjected to the intentions of others, namely those who exert all power, influence and control over the very definition of legal key terms and concepts.
\end{abstract}

\section{Plädoyer für eine kritische Diskussion der Verständlichkeit von Rechtstexten}

Warum soll (wieder einmal) die Verständlichkeit von Rechtstexten nun vor dem Hintergrund eines interaktionsorientierten Ansatzes diskutiert werden, wenn es doch schon aus der eigenen Alltagserfahrung her bekannt sein könnte, daß Leser mit Texten in eine Interaktion treten, daß es Wechselwirkungen zwischen Leserbefindlichkeiten und Text(merkmal)en gibt, und daß daher in der Forschung auch reale Leser und die Wirkweise ihrer Befindlichkeiten auf die Verständlichkeit von Rechtstexten untersucht werden sollten?

* Dipl.-Psych. Christian F.G. Schendera

Mühlingstr. 1

D-69121 Heidelberg

fon: +49 - 6221- 484328

email: heidelberg@method-consult.de 
Zunächst einmal ist die Auffassung einer Leser-Text-Interaktion weder in der Theorie noch in der Praxis zur Rechtsverständlichkeit so selbstverständlich, wie man meinen sollte (Schendera 2000a, Schendera 2000b). Zweitens gibt der übliche Interaktionsbegriff für die Diskussion von Rechtsverständlichkeit vor, daß sich die notwendige Analyse auf die Interaktion zwischen Leser und Text beschränken soll. Die Konzentration auf die bloße Interaktion von Lesern und Texten verstellt jedoch den Blick auf weitere Faktoren, die einen nachhaltigen Einfluß auf das ausüben können, was man unter „Recht“ oder „Verständlichkeit“" verstehen könnte. Dieser Einfluß könnte durchaus ebenfalls als Interaktion beschrieben werden. Zu guter Letzt wird sich zeigen, daß eine mangelnde Konsensualität über einen fundamentalen Terminus wie „Rechtstext“ und in der Folge damit auch „,Rechtsverständlichkeit“" nicht nur ein vermeintlich vernachlässigbares Kommunikationsproblem, sondern ein brisantes Problem der Definition rechtstechnischer Konstrukte ist.

Es wird sich konsequenterweise zeigen, daß die anfangs hilfreiche Verwendung des Interaktionbegriffs ${ }^{1}$ in der Folge der Diskussion der Rechtsverständlichkeit zunehmend unangemessen wird. Der Begriff der „Interaktion“" in seiner abstrakten Grundbedeutung der ,Wechselwirkung“ ist anfangs hinreichend, um auf die Existenz weiterer Interaktionen hinzuweisen. Die verschiedenen Varianten der Interaktion, die zwischen den unterschiedlichsten Ebenen und Faktoren parallel und dynamisch existieren, können jedoch nicht mit einem einheitlichen Interaktionsbegriff

1 Der Begriff der Interaktion ist vielschichtig und interdisziplinär zugleich. Die im folgenden exemplarisch und schlagwortartig aufgelisteten Teilbedeutungen stammen vorrangig aus dem sozialwissenschaftlichen Terminologiekontext. Die Teilbedeutungen reichen von Varianten individueller Auseinandersetzung über kommunikative Handlungen zwischen mindestens zwei Personen bis hin zu technisch-methodischen Spezialbegriffen. Für eine nuancierte Vertiefung alleine des Teilbegriffes „,(soziale bzw. kommunikative) Interaktion" sei exemplarisch auf Graumann (1972) verwiesen. Zu den Varianten individueller Auseinandersetzung zählen z.B. individuelle Interaktionen mit (sozial) relevanten Gegenständen (Personen, Situationen), Einstellungen, Meinungen, oder Wertbezügen. Die (a)symmetrische Wechselbeziehung zwischen mindestens zwei (kommunikativen) Handlungen oder kommunikativ Handelnden zählt zu den Varianten kommunikativer Interaktionen. In der Statistik wird mit Interaktion ein Varianzanteil bezeichnet, der nicht durch den Einfluß der einzelnen unabhängigen Variablen, sondern als Funktion der gemeinsamen Variation von zwei oder mehr unabhängigen Variablen erklärt, wobei diese sich nicht bloß addieren, sondern einander kompensieren oder verstärken (Bortz 1993, Bortz \& Döring 1995). 
beschrieben werden. Eine rezipierende Interaktion zwischen einer Leserin und einem Text ist z.B. eine andere Art der Interaktion, als eine gleichberechtigt-kommunikative Interaktion zwischen zwei Personen in einem sozialen Kontext, eine asymmetrisch-regulative Interaktion zwischen zwei nicht gleichberechtigten Personen oder der nicht akzeptierten regulativen Funktion eines normativen Textes. Ein Interaktionsbegriff gibt eine Einheitlichkeit von Wechselwirkungen vor, die allerdings der Vielfalt und den Unterschieden zwischen den Interaktionsvarianten in Art (siehe auch Fußnote 2), Richtung (Direktionalität), Aktivität (Dominanz), und Berechtigung (Wertigkeit) nicht gerecht wird.

Der folgende Abschnitt wird ausgehend von den üblicherweise untersuchten und diskutierten Varianten einer Leser-Text-Interaktion auf weitere Ebenen und Formen der Interaktion im Zusammenhang mit der Rechtsverständlichkeit übergehen.

\section{2. Über das einfache Modell einer Leser-Text-Interaktion hinaus - Ebenen und Formen der Interaktion im engeren Kontext zur Verständlichkeit}

Am Anfang dieses Abschnitts werden zunächst die Grundelemente einer Leser-Text-Interaktion dargestellt. Im Verlaufe der Elaboration und Ausdifferenzierung dieser Elemente werden die Ebenen und Formen der Interaktionen vorgestellt, die im Zusammenhang mit der Rechtsverständlichkeit auf der Basis von Texten üblicherweise untersucht und diskutiert werden. Im Anschluß daran kann an einem erweiterten Leser-Text-Interaktionsmodell gezeigt werden, welche weiteren Einflußfaktoren, die für die Definition, Untersuchung und Diskussion von Rechtsverständlichkeit bedeutsam sind, bislang weitestgehend aus dem wissenschaftlichen Diskurs ausgeklammert worden sind.

\subsection{Das Grundmodell}

Doch zunächst zurück zum Grundmodell: Welche Elemente müssen in einem Grundmodell der Leser-Text-Interaktion mindestens gegeben sein, um von der Verständlichkeit eines Textes sprechen zu können? Aus unserer alltäglichen Erfahrung wissen wir: Eine LeserIn, ein Text, und eine Beziehung zwischen LeserIn und Text, die in ein bestimmtes Ergebnis mündet. Betrachten wir nun die Elemente LeserIn, Text, Beziehung und 
Ergebnis genauer: Zunächst einzeln, und danach in immer komplexeren Zusammenhängen.

\subsubsection{Leser}

Das Element LeserIn steht für Menschen, die etwas tun, nämlich lesen; diese alltagssprachlich augenscheinlich banale Feststellung sollte sich relativieren, wenn man sich vergegenwärtigt, daß z.B. der Begriff „LeserIn" leserpsychologisch völlig unterschiedlich definiert wird. LeserInnen werden je nach Menschenbildannahme völlig verschiedene Kompetenzen zugeschrieben: Je nach Lern- und Handlungstheorie gibt es aktiv elaborierende, modifizierende, selegierende, oder auch determinierte passive Leser (vgl. Drinkmann/Groeben 1989, Groeben 1986, Groeben 1982, Groeben/Vorderer 1988). Dies bedeutet, daß je nach theoretischem Modell des Lesers nicht nur der Vorgang des Lesens völlig verschieden beschrieben bzw. erklärt wird, sondern letztlich auch das Ergebnis des Lesens, nämlich die Verständlichkeit bzw. das Verstehen von Rechtstexten (Christmann 1989, Graesser/Britton 1994, Kintsch 1998, Kintsch 1985, Kintsch/ van Dijk 1978).

Die Theorien, die diesen Modellen zugrunde liegen, befassen sich u.a. mit intellektuellen Kompetenzen, Befindlichkeiten und Strategien, die zur Konstruktion und Zuweisung von Bedeutung wichtig sind, z.B. die verschiedenen Ebenen der Bewußtheit, die Arten des Lernens, das (inhaltliche ) Ausmaß der Bildung, Motivation, die (formale) Wissensrepräsentation usw. Der Blickwinkel dieser Theorien erlaubt die Frage, ob und inwieweit auch Interaktionen zwischen diesen Kompetenzen, Befindlichkeiten und Strategien im Zusammenhang mit Rechtsverständlichkeit stehen. Die Forschung belegt (siehe Schendera 2000a, Schendera 2000b), daß z.B. Merkmale wie Bildung oder Vorwissen einen Einfluß auf die Verständlichkeit eines Textes haben können.

\subsubsection{Text}

Was ist aber nun ein Text? Zum wissenschaftlichen Begriff des Textes gibt es keine allgemeingültige Definition, sondern Definitionsvarianten, die sich je nach Definiens im einzelnen unterscheiden (u.a. Brinker 2001: 12ff., Vater 1994: 16ff., Linke et al. 2001, Bußmann 1990, De Beaugrande/Dressler 1981: 3ff., Wawrzyniak 1980: 7f.). Der jeweilige Begriff 
des Textes wird dabei meist über mehrere Kriterien der materiellen bzw. strukturellen Form oder kommunikativen Funktion gleichzeitig definiert.

Die (hier: abgeschlossene) materielle Form (z.B. Brief) kann ein Definiens dafür sein, ob z.B. ein Definiendum ein Text ist. Abgeschlossen bedeutet hier, daß mit den Grenzen der Form (z.B. Anfang und Ende eines Briefes) auch der Text selbst begrenzt wird. Die Grenzen der Form sind die Grenzen des Textes.

Die Definiens hinsichtlich der Funktion eines Textes setzen keinen abgeschlossenen Text voraus. Definiens zur Funktion eines Textes können z.B. sein: Die Fixierung bzw. Vermittlung immaterieller Inhalte (z.B. „Buchstabe des Gesetzes“), die Steuerung komplexer politischer Phänomene (z.B. Rechts- und Verwaltungsvorschriften), oder auch als Projektionsfläche potentiell polysemer, polyfunktionaler, oder polyvalenter (Rechts)Interpretationen (sensu Hauptmeier/Schmidt 1985). Die Funktion eines Textes wird z.B. wird nicht notwendigerweise durch die Grenzen seiner Form beschränkt: Die Grenzen der Form sind nicht die Grenzen der Funktion.

Das Kriterium der (Un)Abgeschlossenheit rückt die Diskussion des Begriffs des Textes in die Nähe der Interaktion (Brinker 2001, Bußmann 1990): Wird ein Text sprachsystematisch-grammatisch als abgeschlossen definiert, so wird ein Text als autark gesehen, durch seine Formgrenzen isoliert von jedem außertextlichen Kontext. Die oberste Analyseeinheit ist der Text selbst, die hierarchisch nächsten üblicherweise Satz bzw. Phrase usw. (zu zusätzlichen Einheiten siehe Schweikle/Schweikle 1990: 232).

Merkmale für Text(un)verständlichkeit sind dabei auf den Text beschränkt, so wie er durch die Grenzen seiner materiellen Form definiert wird, also u.a. Satzlänge, das Ausmaß der Satzverschachtelung, oder auch technisches Vokabular. Wird ein Text implizit oder explizit als abgeschlossen definiert, so sind Faktoren außerhalb der Formgrenzen (z.B. kommunikative Kontexte, dominante Gruppen, oder auch funktionale Definiens) nicht verantwortlich für eine Rechts(un)verständlichkeit.

Texte sind jedoch niemals absolut hermetisch geschlossene sich selbst erschaffende und ausschließlich auf sich selbst rückbeziehende Strukturund Bedeutungssysteme, sondern sind immer in kommunikative Situationen bzw. Sprachhandlungen von Textproduzenten bzw. -rezipienten eingebettet (Brinker 2001: 15; Vater 1994: 15, Bußmann 1990: 776). 
Textstrukturen sind, ebenfalls wie der Textinhalt, prinzipiell offen für Interpretationen seitens kommunikativ Handelnder in ihren jeweiligen Kontexten. Dieser Befund rückt das Phänomen Text in die Nähe der Kommunikation (Bußmann 1990: 779: „Kommunikative Einheit“, De Beaugrande/Dressler 1981: 3: „Kommunikative Okkurrenz“) und auch Kognition (Vater 1994: 65, Schwarz 1992: 159ff., de Beaugrande/ Dressler 1981: 88ff., Rickheit/Strohner 1993). Brinker (2001: 17) definiert z.B. das Phänomen „Text“ als „,eine begrenzte Folge von sprachlichen Zeichen, die in sich kohärent ist und die als Ganzes eine erkennbare kommunikative Funktion signalisiert.“

Ist ein Text als offen definiert, so hat dies mehrere bedeutsame Konsequenzen:

Der offene Text ist nicht notwendigerweise ausschließlich die oberste Analyseeinheit, sondern kann je nach Perspektive und Forschungsinteresse auch eine nachgeordnete Analyseeinheit sein. Ist ein Text eine nachgeordnete Analyseeinheit (z.B. im Hinblick auf Faktoren wie z.B. Dominanz, Komplexität, Intentionalität (Vater 1994: 51, De Beaugrande/Dressler 1981: 8ff.) und Perspektivität (Hartung 1997), so ist er den Kontexten und den darin befindlichen LeserInnen und ihren Eigenschaften nachgeordnet. Das heißt, zu den bereits genannten intellektuellen Kompetenzen etc. treten nun Faktoren, die im Kontext der Interaktion mit einem und um einen Text wirksam werden, z.B. individuelle kommunikative Kompetenzen, aber auch der Einfluß von Gruppierungen (z.B. Lobbies), oder hochkomplexe sozioregulative Strategien oder Mechanismen, z.B. um Macht, Einfluß, oder Vorstellungen davon, „was recht ist" (oder auch nicht).

Ein Text wird nicht mehr als eine invariante und (nach außen) hermetisch geschlossene Struktur- und Bedeutungseinheit betrachtet; statt dessen wird die Analyseperspektive auf den jeweiligen institutionellen, kommunikativen, und kognitiven etc. Kontext erweitert, innerhalb dessen sich ein Text befindet, nämlich seinen Einfluß auf die Produktion und Rezeption des Textinhalts, und damit auch der stillschweigenden Definition dessen, was denn je nach Kontext unter einem Text zu verstehen sei. Offen definierte Texte sind oft in mehrere Kontexte zugleich eingebettet, und sind daher immer auch ein dynamisches Produkt solcher mehrdimensionaler Interaktionen, z.B. individueller Kognitionen, gruppenspezifischer Leser-Text-Interaktionen, soziohistorisch gewachsener kommuni- 
kativer (oder auch anderer) Interaktionen innerhalb und zwischen den jeweiligen LeserInnen-Kontexten in dem Sinne, ,,daß Texte immer eingebettet sind in eine Kommunikationssituation, daß sie immer in einem konkreten Kommunikationsprozeß stehen, in dem Sprecher und Hörer bzw. Autor und Leser mit ihren sozialen und situativen Voraussetzungen und Beziehungen die wichtigsten Faktoren darstellen“ (Brinker, 2001: $15)$.

Kommen wir zurück zu den einzelnen Elementen einer LeserIn-TextBeziehung. Wir hatten bis jetzt LeserIn und Text, es fehlen noch die Elemente Beziehung und Ergebnis.

\subsubsection{Leser-Text-Beziehung (Interaktion)}

Eine Beziehung zwischen LeserIn und Text aktiv zu gestalten, ist nur LeserInnen möglich. Je nach herangezogener Kompetenzdefinition von „LeserIn“ folgt daraus ein jeweils anders gestalteter Umgang mit einem Text. In Abhängigkeit der (in den Menschenbildannahmen theoretisch) unterstellten Kompetenzen kann eine solche LeserIn-Text-Beziehung aktiv-elaborativ, reduktiv-modifizierend oder selektiv-reaktiv gestaltet sein (diese „Bindestrich“-Begrifflichkeiten beschreiben graduelle Unterschiede in der Selbständigkeit und der Komplexität des Umgangs mit Texten, siehe z.B. Groeben 1982).

Die Beziehung zwischen Text und LeserIn kann vom Text nur passiv mitgestaltet werden. Nur dann, wenn eine LeserIn als passiv definiert ist, können Text-Merkmale dominieren. Eine solche Text-Leser-Beziehung wird als passiv-determiniert bezeichnet (vgl. Drinkmann/Groeben 1989, Groeben 1986, Groeben 1982, Groeben/Vorderer 1988).

Die jeweilige Dominanz der Leser-Eigenschaften und Text-Merkmale ist also entscheidend für die Gestaltung der Beziehung zwischen Leser und Text, und damit in der Folge für ihr Ergebnis, die Verständlichkeit bzw. das Verstehen.

Ich habe das Ergebnis einer Leser-Text-Beziehung der Einfachheit halber an einem als geschlossen definierten Text dargestellt (auf eine kommunikationsorientierte Diskussion komme ich später nochmals zurück); zugleich läßt sich damit aber auch zeigen, wie absurd die Vorstellung eines geschlossenen Textes ist. Sobald ein Leser in eine Interaktion mit einem Text tritt, ist dieser Text kein geschlossener bzw. 
invarianter mehr. Dies gilt vorrangig für die Textsemantik und -pragmatik, aber auch für die Textsyntaktik (Schendera, 2000a, 2000b); es bleiben zwar die Textstrukturen als solche invariant, nicht jedoch ihre semantikgeleitete referentielle Neuinterpretation, d.h. semantische Neuorganisation.

Innerhalb von Leser-Text-Beziehungen stößt der eingangs eingeführte geschlossene Textbegriff an seine Grenzen, möchte man sagen; vielleicht könnte man aber auch so weit gehen und formulieren: Innerhalb von Leser-Text-Beziehungen wird jeder geschlossene Text zu einem offenen Text.

\subsubsection{Ergebnis (Rechtsverständlichkeit)}

Das noch fehlende Element Ergebnis, in unserem Zusammenhang also Rechtsverständlichkeit ( auf den Begriff werde ich an dieser Stelle aus guten Gründen nicht weiter eingehen, auch nicht auf die Abgrenzung zu Verstehen), ist immer eine Folge der (mindestens) drei anderen Elemente LeserIn, Text und Beziehung. Dasselbe Ergebnis (in der Realität) kann dabei je nach Theorie immer anders beschrieben und erklärt werden. Nämlich je nach dem, wie über Vorannahmen zu vermuteten LeserInEigenschaften die Beziehung zwischen LeserIn und Text (z.B. Kompetenzen) und Text-Merkmale definiert wird. Umgekehrt wird praktisch das Ergebnis immer ein anderes sein, wenn die einzelnen Elemente dieser komplexen Beziehung variiert werden, also wenn z.B. LeserInnen mit hoher Bildung mit einfachen Texten oder LeserInnen mit geringerer Bildung mit komplexeren Texten konfrontiert werden, usw.

Die Verständlichkeit eines Textes kann erst während einer Interaktion festgestellt werden, oder alternativ auf der Basis eines Kriterienkatalogs, der nach einer Interaktion mit einer oder mehreren LeserInnen angelegt wurde. Die Verständlichkeit eines Textes ist der Interaktion mit einer LeserIn immer zeitlich bzw. kausal nachgeordnet. Verständlichkeit einem Text zu attestieren, ohne ihn gelesen zu haben, ist reine Spekulation.

Wie zu sehen ist, sind Text-Leser-Beziehungen höchst komplexe und dynamische Gewebe. Es lassen sich mehrere grundsätzliche Interaktionsebenen identifizieren: Zunächst einmal zwischen LeserIn und Text und dann verfeinert zwischen LeserIn-Eigenschaften, z.B. Bildung und einem Text, und auch die Wechselwirkung einzelner Text-Merkmale mit Verständlichkeit, z.B. Satzlänge. Wie bereits erwähnt, finden z.B. LeserInnen 
mit höherer Bildung Rechtstexte verständlicher als LeserInnen mit niedrigerer Bildung.

Theoretisch und empirisch sind einige Lücken zu konstatieren. Auf den sprachsystematischen Textbegriff bezogen bedeutet dies, daß z.B. noch nicht untersucht wurde, ob LeserInnen mit denselben Kompetenzen immer auch dieselben Text-Merkmale als (un)verständlichkeitsevozierend finden bzw. ob LeserInnen mit unterschiedlichen Kompetenzen (z.B. Bildung) verschiedene Text-Merkmale als (un)verständlichkeitsevozierend beurteilen. Von kumulierten Effekten mehrerer LeserInnen-Eigenschaften bzw. der Kombinatorik von Merkmalen innerhalb von Texten ganz zu schweigen.

An dieser Stelle möchte ich nun auf den eingangs eingeführten kommunikationsorientierten Text-Begriff zurückgehen, der auch den Kontext um einen Text berücksichtigt.

Unser aller Wissen um Leser-Text-Interaktionen stammt selten aus direkt beobachteten, sondern vorwiegend aus kommunikativ vermittelten Ergebnissen des Kontexts ,Verständlichkeitsforschung“. Das Besondere am Kontext „Verständlichkeitsforschung“ ist nun nicht, daß er durch Theoriebildung, Forschungs- und Publikationspraxis das Wissen über Verständlichkeit definiert, und daß er darin durch (sub)optimale Umsetzung auch mehr oder weniger erfolgreich ist (Schendera 2001a, Schendera 2000a, Schendera 2000b). Das Bedeutsame ist vor allem, daß innerhalb dieses Kontexts stillschweigend der Begriff des geschlossenen Textes verwendet wird: Entweder sind nur allein der Text und seine Merkmale (Inhalte, Strukturen) für seine (Un)Verständlichkeit verantwortlich, oder allein die Leserkompetenzen, die in ihrer Wechselwirkung mit diesem Text und seinen Merkmalen (nicht) zurecht kommen.

Auch wenn dies im Detail zutreffen mag, blendet diese in der operationalen Umsetzung der Forschungspraxis hochkomplexe, aber dem Forschungsgegenstand „Rechtsverständlichkeit“ unangemessen vereinfachende Ansicht aus, daß ein Text eben nicht nur in reiner rechtsfixierender Funktion verwendet wird, also als geschlossener Rechtstext, sondern vor allem auch den Einflu $\beta$ anderer Kontexte auf die Rechtsverständlichkeit auf der Basis eines offenen Rechtstextes.

Zur Orientierung an dieser Stelle ein kurzes Zwischenresümee: Die ursprüngliche ,reine“ Interaktion zwischen LeserIn und Text wurde von der Ebene relativ globaler „Schlagworte“ in immer feinere Konstrukte 
differenziert. Das Leser-Text-Modell wurde dann in einen Kontext (hier: Forschung) platziert, der sich v.a. dadurch auszeichnet, daß er eine ziemlich vereinfachende Auffassung vom Textbegriff hat. Verschärfend kommt hinzu, daß innerhalb dieses Forschungskontexts nicht alle Ansätze zur Abbildung einer Interaktion tatsächlich auch interaktionsorientiert sind.

Wesentlich jedoch ist die explizite Berücksichtigung eines Kontextes, also textferner Einflüsse, auf die Rechtsverständlichkeit: Sei es der Forschungskontext selbst (z.B. in kritischer Selbstreflexion seiner Rolle innerhalb des Vorgangs der Wissenskonstruktion i.S.e. VorannahmenMethoden-Gegenstand-Interaktion), sei es in den individuellen Überzeugungen von Publikationsautoritäten (z.B. in Gestalt unbegründeter oder einseitiger Skeptizismen, sog. Bias), sei es die Berücksichtigung eines anderen Kontexts (z.B. einer Institution, die u.U. völlig abweichende Annahmen über die Rolle des Textes hat, z.B. nicht in rechtsfixierender, sondern in rein symbolisch repräsentierender Funktion), und noch weitergehend, einer (oder mehrerer) Interaktionen zwischen diesen (oder auch anderen involvierten) Kontexten.

\subsubsection{Gebrauch und Mißbrauch des Textbegriffes und Rechtsverständlichkeit als Folge}

Ein Textbegriff, der in vielen Interaktionsdimensionen eingebettet sein kann, hat zur Folge, daß der Begriff der Verständlichkeit vor allem beim Rechtstext nicht nur zu Mißverständnis, sondern auch zu Mißbrauch führen kann, wenn seine jeweils spezifische Definition und Verwendung im Kontext von Einfluß, Macht, und Kontrolle nicht explizit geklärt wird.

Falls Angehörige verschiedener Kontexte nur mit Mühe oder gar nicht über den Gegenstand „Text“ miteinander kommunizieren können, mag dies eventuell als ein Hinweis auf implizite bzw. nur heuristisch verwendete Textdefinitionen zu sehen sein; ebenfalls nicht abwegig ist die Möglichkeit, daß sich die in den jeweiligen Kontexten verwendeten Textdefinitionen darüber hinaus völlig unterscheiden, was besonders in Wissenschaftskontexten naheliegend ist. Klärung verschafft nur eine interdisziplinäre Interaktion, eine Art gemeinsames Erarbeiten von Inhalt, Struktur und Funktion des Gegenstands „Rechtstext“, um mindestens eine erfolgreiche Kommunikation über diesen Gegenstand zu ermöglichen. Ein differenziertes und beiderseits akzeptiertes Begriffskorpus 
ist die notwendige Grundlage für die erfolgreiche Etablierung und konstruktive Weiterentwicklung von kommunikativem Handeln; falls die Teilnehmer (unter denkbaren Umständen) nicht bereit und fähig sind, ihren Begriffskorpus zu differenzieren, hat dies eine gestörte Interaktion zur Folge, was im Zusammenhang mit ,Rechtstexten und Rechtsverständlichkeit" nicht nur wissenschaftliches(politisches), sondern auch (rechts)politisches Handeln unmöglich machen wird.

Das Definieren der Begriffe „Rechtstext“ und „Rechtsverständlichkeit“ durch Einzelpersonen, exklusive Gruppen oder Kontexte im Einzugsbereich von Einfluß, Macht und Kontrolle ist eine undemokratische Festsetzung einer Definition einschließlich all ihrer Konsequenzen. Eine derartige Setzung geht von einem für andere unhinterfragt vorausgesetzten Konsens aus, nämlich daß die von exklusiven Einzelnen (Personen, Gruppen, Kontexte) gesetzten verständlichkeitsrelevanten Definitionen interaktionell bzw. interdisziplinär zustandegekommen, gesellschaftlich allgemein zu akzeptieren, und nicht hinsichtlich Setzung, Inhalt und Funktionalität zu hinterfragen seien. Gerade solche, auf einer undemokratischen Basis gründende aber kollektiv-normativ gelten sollende Festsetzungen, binden an Begriffe wie „Rechtssprache“, „Rechtstext“ und „Rechtsverständlichkeit“ nicht nur besondere Bedeutungen, Auslegungsprinzipien und Handlungsrechtfertigungen, sondern auch stillschweigend ademokratische Annahmen über politische Partizipation (vgl. Dascal/Wroblewski 1991: 440ff.).

Menschen wären in dieser Konstellation immer den Intentionen anderer unterworfen und zwar derjenigen, die die alleinige Macht, Einfluß und Kontrolle über die Definition von Begriffen wie z.B. „Rechtsverständlichkeit“" und ,Rechtstext“" ausüben. ${ }^{2}$

\section{Zusammenfassung und Ausblick}

Dieser Aufsatz versuchte zu zeigen, daß die Rechtsverständlichkeit ein Begriff ist, der zahlreiche Interaktionen und ihre komplexe Verwobenheit in sich birgt. Nur ein interaktionsorientierter Ansatz wäre m.E. in der Lage, diese Komplexität transparent zu machen, daraus konkrete Konsequenzen für die Definition und Verbesserung der Verständlichkeit von Rechtstexten abzuleiten, und in die Praxis verbesserter Rechtsverständlichkeit umzusetzen.

2 Die sprachkonzeptionellen Bemühungen der Rechtstheorie vor allem zu Anfang des III. Reiches sind aufschlußreich (Schendera 1999). 
Rechtstexte stehen inmitten der Spannung und Regulation antagonistisch-pluraler Interessen. Rechtstexte sind genuin politische Texte (Heinemann/Viehweger 1991: 148ff.), von der Produktion, bis hin zur Rezeption.

Rechtsverständlichkeit ist immer auch in die Kontexte Einfluß, Macht, und Kontrolle eingebettet. Die Definition von Rechtsverständlichkeit ist eine politische Handlung, weil in der Folge über die Anwendung von Regeln und Methoden. Rechtsverständlichkeit ist daher immer auch ein politischer Begriff.

Die Festlegung dessen, was man unter Rechtsverständlichkeit zu verstehen hat, ist jedoch keine exklusive Angelegenheit. Eine demokratische Definition von Rechtsverständlichkeit kann nur in einem ausdrücklich freiheitlich-demokratischen Diskurskontext erfolgen. Im Rahmen einer kommunikativen, rationalen und argumentativ-integrativen Interaktion möglichst gleichberechtigter Einzelpersonen, Gruppen und Disziplinen ist eine erschöpfende und systematische Klärung, Prüfung, und Differenzierung der virulenten Begrifflichkeiten, Regelwerke und Konstrukte vorzunehmen, z.B. auch hin auf individuelle Intentionen, oder Gruppeninteressen etc. Jede exklusive Definition und Verwendung von Termini ist explizit und argumentativ zu begründen.

Und es gibt viel zu klären, sehr viel: Internationale Rechtssysteme (USA, UK, EU) und ihre jeweilige Auffassung von „Rechtsverständlichkeit"als Folge von Textarbeit, Interaktionen zwischen internationalen Sprachsystemen (z.B. ,,arabisch“, ,,chinesisch“, ,,westlich“) und „Rechtsverständlichkeit“" Interaktionen zwischen internationalen und nationalen Rechtssystemen und die Folge der Interaktionen zwischen hierarchischen „Rechtstexten“ auf ihre Verständlichkeit (z.B. EU, Schendera, 2001b), Interaktionen innerhalb und zwischen verschiedenen Disziplinen bei der Definition von „Rechtsverständlichkeit“ und natürlich „Rechtsverständlichkeit" als Folge der Interaktion verschiedener Verstehensebenen mit (u.U. verschiedenen Sorten von) Rechtstexten. Auch daß es innerhalb eines Systems hierarchisch oder kontextuell verschieden verständliche Sorten von Rechtstexten gibt (z.B. StVO-Vorschriften vs. Grundgesetze, oder Zivil- vs. Strafrecht) und damit eine kontextspezifische Auffassung von Rechtsverständlichkeit ist doch eigentlich auch kein Problem der Textsorte, oder der Verständlichkeit als solcher, sondern das Phänomen einer adaptiv-flexiblen Auffassung von Verständlichkeit. 
Eine adäquate Erforschung all dieser Phänomene setzt voraus, daß alle Teilnehmer klären, ob die in einer Interaktion verwendeten Begriffe tatsächlich auch dasselbe Phänomen meinen. Falls die Teilnehmer (unter denkbaren Umständen) nicht bereit und fähig sind, ihren Begriffskorpus zu differenzieren, hat dies eine gestörte Interaktion zur Folge, was im Zusammenhang mit „Rechtstexten und Rechtsverständlichkeit“ nicht nur wissenschaftliches(politisches), sondern auch (rechts)politisches Handeln unmöglich machen wird.

Wenn Menschen auf diese Weise an der Definition und Anwendung der Rechtsverständlichkeit partizipieren, folgen sie nicht dem Willen einiger weniger, sondern bewahren ihre Selbstbestimmung in einem freiheitlich-demokratischen Kontext der Interaktion.

\section{Literatur}

Beaugrande, Robert A. de \& Dressler, Wolfgang U. (1981). Einführung in die Textlinguistik. Tübingen: Niemeyer.

Bortz, Jürgen (1993). Statistik für Sozialwissenschaftler. Heidelberg: Springer. Fourth Edition.

Bortz, Jürgen \& Döring, Nicola (1995). Forschungsmethoden und Evaluation. Heidelberg: Springer. Second Edition.

Brinker, Klaus (2001). Linguistische Textanalyse. Eine Einführung in Grundbegriffe und Methoden. Berlin: Erich Schmidt. Fifth edition.

Britton, Bruce K. \& Graesser Arthur C. (1996). Five metaphors for text understanding. In Britton, B.K. \& Graesser, A.C. (eds.) (1996). Models of Understanding Text, Mahwah NJ: Lawrence Erlbaum 341-351.

Bußmann, Hadumod (1990). Lexikon der Sprachwissenschaft. Stuttgart: Kröner.

Christmann, Ursula (1989). Modelle der Textverarbeitung: Textbeschreibung als Textverstehen. Münster: Aschendorff.

Dascal, Marcelo \& Wroblewski, Jerzy (1991). The rational law-maker and the pragmatics of legal interpretation. In Journal of Pragmatics 15 (5), 421-444.

Drinkmann, Arno \& Groeben, Norbert (1989). Metaanalysen für Textwirkungsforschung: Methodologische Varianten und inhaltliche Ergebnisse im Bereich der Persuasionswirkung von Texten. Weinheim: Deutscher Studienverlag.

Graumann, Carl F. (1972). Interaktion und Kommunikation. In Gottschaldt, K. et al. (eds.) (1972). Handbuch der Psychologie (Vol. 7.: Sozialpsychologie). Göttingen: Hogrefe. 1109-1262.

Groeben, Norbert (1982). Leserpsychologie: Textverstehen - Textverständlichkeit. Münster: Aschendorff. 


\section{8}

Groeben, Norbert (1986). Handeln, Tun, Verhalten. Tübingen: A.Francke.

Groeben, Norbert \& Vorderer, Peter (1988). Leserpsychologie: Lesemotivation Lektürewirkung. Münster: Aschendorff.

Hartung, Wolfgang (1997). Text und Perspektive: Elemente einer konstruktivistischen Textauffassung. In Antos, G. \& Tietz, H. (eds.) (1997). Die Zukunft der Textlinguistik: Traditionen, Transformationen, Trends. Tübingen: Max Niemeyer. 13-25.

Hauptmeier, Helmut \& Schmidt, Siegfried J. (1985). Einführung in die Empirische Literaturwissenschaft. Braunschweig/Baden: Vieweg.

Heinemann, Wolfgang \& Viehweger, Dieter (1991). Textlinguistik: Eine Einführung. Tübingen: Niemeyer.

Isenberg, Horst (1976). Einige Grundbegriffe für eine linguistische Texttheorie. In Danes, F. \& Viehweger, D. (1976) (eds.) Probleme der Textgrammatik I. Berlin: Akad. Verlag.

Kintsch, Walter (1998). Comprehension: A Paradigm for Cognition. Cambridge: Cambridge University Press.

Kintsch, Walter (1985). Text processing: A psychological model. In van Dijk, T.A. (1985) (ed.) Handbook of Discourse Analysis, Vol. 1: Disciplines of Discourse. London/ New York: Academic Press. 231-243.

Kintsch, Walter \& Dijk, Teun A. van (1978). Toward a model of text comprehension and production. In Psychological Review 85 (5) 363-394.

Linke, Angelika, Nussbaumer, Markus \& Portmann, Paul R. (2001): Studienbuch Linguistik (Reihe Germanistische Linguistik Bd. 121). Tübingen: Niemeyer. Fourth edition.

Rickheit, Gert \& Strohner, Hans (1993). Grundlagen der kognitiven Sprachverarbeitung. Modelle, Methoden, Ergebnisse. Tübingen: Francke.

Schendera, Christian FG (1999). Constructing definitions to prosecute the political enemy: National socialist legal theorists and ,their' language. Unveröffentlichter Vortrag auf der Critical Legal Congress, 18.09.1999 (London, UK ).

Schendera, Christian FG (2000a). Die Erforschung der Verständlichkeit von normativen Texten: Eine kritische Darstellung von Modellen, Methoden und Ergebnissen. In Zeitschrift für Sprachwissenschaft, 19.1, 3-33.

Schendera, Christian FG (2000b). Die Verständlichkeit normativer Texte: eine kritische Darstellung der Forschungslage. In LeGes - Gesetzgebung \& Evaluation (CH), 2, 99-134.

Schendera, Christian FG (2001a). Der Nutzen wissenschaftlicher Verfahren zur Verbesserung der Verständlichkeit von Gesetzestexten: Überblick, ausgewählte Beispiele, Diskussion. Unveröffentlichter Vortrag im Rahmen des Workshops „Sprache des Rechts. Vermitteln, Verstehen, Verwechseln“ am 22./24.02.2001 auf Einladung der Berlin-Brandenburgischen Akademie der Wissenschaften, Schloß Blankensee, Berlin.

Schendera, Christian F.G. (2001b). Verständlichkeit von Rechtstexten und ihre Optimierung. Beitrag zur Internationalen Fachkonferenz „,Deutsch im vielsprachigen 
Europa“ am 26./27.11.2001 in Brüssel, auf Einladung der Gesellschaft für Deutsche Sprache, Wiesbaden.

Schmidt, Siegfried J. (1973). Texttheorie. Probleme einer Linguistik der sprachlichen Kommunikation. München: Fink.

Schweikle, Günther \& Schweikle, Irmgard (1990). Metzler-Literatur-Lexikon: Begriffe und Definitionen. Stuttgart: Metzler. Second edition.

Vater, Heinz (1994). Einführung in die Textlinguistik: Struktur, Thema und Referenz in Texten. München: UTB Wissenschaft.

Wawrzyniak, Zdzislaw (1980). Einführung in die Textwissenschaft: Probleme der Textbildung im Deutschen. Warszawa: Panstwowe Wydawn. 
